\title{
BMJ Open Diet and physical activity in pregnancy to prevent gestational diabetes: a protocol for an individual participant data (IPD) meta-analysis on the differential effects of interventions with economic evaluation
}

Dyuti Coomar, ${ }^{1}$ Jonathan M Hazlehurst (D) , ${ }^{2}$ Frances Austin, ${ }^{3}$ Charlie Foster, ${ }^{4}$ Graham A Hitman, ${ }^{5}$ Nicola Heslehurst, ${ }^{6}$ Stamatina lliodromiti, ${ }^{7}$ Ana Pilar Betran, ${ }^{8}$ Ngawai Moss, ${ }^{9}$ Lucilla Poston, ${ }^{10}$ Krishnarajah Nirantharakumar, ${ }^{1}$ Tracy Roberts, ${ }^{11}$ Sharon A Simpson, ${ }^{12}$ Helena J Teede, ${ }^{13}$ Richard Riley, ${ }^{14}$ John Allotey, ${ }^{1,15}$ Shakila Thangaratinam, ${ }^{2,16}$ On behalf of the International Weight Management in Pregnancy (i-WIP) Collaborative Group

To cite: Coomar D, Hazlehurst JM, Austin F, et al. Diet and physical activity in pregnancy to prevent gestational diabetes: a protocol for an individual participant data (IPD) meta-analysis on the differential effects of interventions with economic evaluation. BMJ Open 2021;11:e048119. doi:10.1136/ bmjopen-2020-048119

- Prepublication history and additional material are published online only. To view please visit the journal online (http://dx.doi. org/10.1136/bmjopen-2020048119).

Received 19 December 2020 Revised 15 March 2021 Accepted 16 March 2021

Check for updates

(C) Author(s) (or their employer(s)) 2021. Re-use permitted under CC BY-NC. No commercial re-use. See rights and permissions. Published by BMJ.

For numbered affiliations see end of article.

Correspondence to Dr Jonathan M Hazlehurst; j.m.hazlehurst@bham.ac.uk

\section{ABSTRACT}

Introduction Mothers with gestational diabetes mellitus (GDM) are at increased risk of pregnancy-related complications and developing type 2 diabetes after delivery. Diet and physical activity-based interventions may prevent GDM, but variations in populations, interventions and outcomes in primary trials have limited the translation of available evidence into practice. We plan to undertake an individual participant data (IPD) meta-analysis of randomised trials to assess the differential effects and cost-effectiveness of diet and physical activity-based interventions in preventing GDM and its complications. Methods The International Weight Management in Pregnancy Collaborative Network database is a living repository of IPD from randomised trials on diet and physical activity in pregnancy identified through a systematic literature search. We shall update our existing search on MEDLINE, Embase, BIOSIS, LILACS, Pascal, Science Citation Index, Cochrane Database of Systematic Reviews, Cochrane Central Register of Controlled Trials, Database of Abstracts of Reviews of Effects and Health Technology Assessment Database without language restriction to identify relevant trials until March 2021. Primary researchers will be invited to join the Network and share their IPD. Trials including women with GDM at baseline will be excluded. We shall perform a one and two stage random-effect meta-analysis for each intervention type (all interventions, diet-based, physical activity-based and mixed approach) to obtain summary intervention effects on GDM with 95\% Cls and summary treatment-covariate interactions. Heterogeneity will be summarised using $\mathrm{I}^{2}$ and $\mathrm{tau}^{2}$ statistics with $95 \%$ prediction intervals. Publication and availability bias will be assessed by examining small study effects. Study quality of included trials will be assessed by the Cochrane Risk of Bias tool, and the Grading of Recommendations,

\section{Strengths and limitations of this study}

- The individual participant data (IPD) meta-analysis builds on an established global group's (International Weight Management in Pregnancy) living database (24766 women, 58 trials) with the potential to expand further with additional published data.

- Increased power in IPD compared with aggregate meta-analysis to determine the differential effects of lifestyle interventions on gestational diabetes.

Detailed mapping of the of the intervention components will enable translation of the results to clinical practice.

- Limitations include potential lack of access to new data and missingness in available data.

Assessment, Development and Evaluations approach will be used to grade the evidence in the results. A modelbased economic analysis will be carried out to assess the cost-effectiveness of interventions to prevent GDM and its complications compared with usual care.

Ethics and dissemination Ethics approval is not required. The study is registered on the International Prospective Register of Systematic Reviews (CRD42020212884). Results will be submitted for publication in peer-reviewed journals.

\section{BACKGROUND}

Gestational diabetes mellitus (GDM) refers to glucose intolerance that is first diagnosed in pregnancy. ${ }^{1}$ GDM affects between $7 \%$ and $27 \%$ of pregnancies globally with variations in estimates according to the population and criteria used for diagnosis. ${ }^{2}$ During 
pregnancy, mothers with GDM are at increased risk of pre-eclampsia, caesarean section and preterm delivery; their babies are at risk of shoulder dystocia, hyperbilirubinaemia, neonatal hypoglycaemia and of being admitted to neonatal intensive care. ${ }^{3}$ GDM is estimated to increase the cost of maternity care alone by $34 \%-95 \%$ compared with women without GDM. ${ }^{4}$ Women with a history of GDM are at 10-fold higher risk of developing type 2 diabetes mellitus with a cumulative incidence of around $16 \% .{ }^{6}$ Babies born to women with GDM are also at risk of obesity ${ }^{7}$ and type 2 diabetes $^{8}$; the long-term complications in the mother and baby have a significant effect on their life expectancy ${ }^{9}$ and quality of life. ${ }^{10}$ The UK's National Health Service (NHS) spend on type 2 diabetes is projected to increase from $£ 8.8$ to over $£ 13$ billion per year in the next 15 years. ${ }^{11}$

Despite the publication of over 70 trials to date on the effects of lifestyle interventions on gestational diabetes, with over $£ 10$ million invested, the findings have not been translated into clinical practice, guidelines and policy frameworks. ${ }^{12-14}$ Meta-analyses of studies on diet and physical activity-based interventions in pregnancy have shown a reduction in rates of GDM than usual care, but studies varied in population, intervention and outcome definitions. ${ }^{14}$ We also do not know if the beneficial effects of diet and physical activity apply to all pregnant women or only to subgroups of women with risk factors such as high Body Mass Index (BMI), high maternal age, ethnic minority origin and low socioeconomic status. The implementation of lifestyle interventions in a scalable way is also hindered by the lack of details about the type, intensity and setting of the effective interventions, and the costeffectiveness of the intervention.

We plan to undertake an individual participant data (IPD) meta-analysis of diet and physical activity-based interventions to assess the intervention effects on GDM and to assess if the effects vary according to maternal characteristics including age, BMI, parity, ethnicity and socioeconomic status.

\section{Objectives}

Primary

1. To evaluate the effects of diet and physical activitybased lifestyle interventions in pregnancy, across all interventions, and for each type of intervention (dietbased, physical activity-based and mixed) on gestational diabetes as defined by the National Institute for Health and Care Excellence (NICE) and by study authors.

2. To assess the differential effects of interventions according to the maternal characteristics (BMI, age, parity, ethnicity and socioeconomic status) on gestational diabetes.

\section{Secondary}

1. To evaluate the effects of the interventions on critically important (1) maternal complications such as hypertensive diseases, caesarean section or preterm birth; and (2) offspring complications such as stillbirth, large for gestational age or admission to the neonatal unit in women with gestational diabetes.

2. To categorise the interventions by core components and to undertake network meta-analysis to rank them by effectiveness.

3. To assess the effects of interventions for specific other definitions of gestational diabetes (WHO, International Association of Diabetes and Pregnancy Study Group (IADPSG), modified IADPSG, and American Diabetes Association (ADA)), and on fasting and 2-hour postprandial glucose levels.

4. To determine the cost-effectiveness of interventions using decision-analytical modelling.

\section{METHODS/DESIGN}

The protocol adheres to Preferred Reporting Items for Systematic Review and Meta-Analysis Protocols reporting statement ${ }^{15}$ and has been registered on the International Prospective Register of Systematic Reviews with registration ID CRD42020212884, and our IPD meta-analytical approach will follow existing methodological guidelines and adhere to the Preferred Reporting Items for Systematic Reviews and Meta-Analyses IPD reporting statement. ${ }^{16}$

\section{Patient and public involvement}

Patients have been involved with this work throughout and have informed design, outcome selection and reporting.

\section{Literature search}

We will update the literature search using our existing search strategy ${ }^{14}$ to identify new trials that have been published since the completion of our previous review. ${ }^{12}$ We will search MEDLINE, Embase, BIOSIS, LILACS, Pascal, Science Citation Index, Cochrane Database of Systematic Reviews, Cochrane Central Register of Controlled Trials, Database of Abstracts of Reviews of Effects and Health Technology Assessment Database without language restrictions up to March 2021. Two independent reviewers will extract data in duplicate.

\section{Establishment of the International Weight Management in Pregnancy (i-WIP) group}

Our i-WIP collaborative group was established in 2013 (40 researchers, 16 countries), which brings together researchers of primary trials on diet and physical activity in pregnancy who have shared IPD from their trials towards the collaborative group database, forming the largest living global database on diet and physical activity interventions in pregnancy. The group is supported by the WHO, and to date, we have access to 24766 women from 58 studies (online supplemental appendix 1).

\section{Inclusion and exclusion criteria}

Trials with random allocation (individual or cluster) on diet and physical activity-based interventions in pregnancy compared with standard antenatal care will be eligible for inclusion. Any trials that included women 
at baseline with gestational diabetes will be excluded. We will only include pregnant women with a BMI of $\geq 18.5 \mathrm{~kg} / \mathrm{m}^{2}$ in early pregnancy to exclude women who are underweight. We will evaluate three main interventions for gestational diabetes prevention in pregnancy: diet-based, physical activity-based, and mixed approach interventions incorporating diet and physical activity components underpinned by behavioural approach. Diet-based interventions include various dietary patterns such as Mediterranean-style diet, low calorie diet and low glycaemic index diet, which are offered by clinicians, dietitians, physiotherapists, or commercial companies in both primary and secondary care settings. The interventions are delivered using vehicles such as print or digital media, phone, face-to-face meetings in either one-on-one or group sessions, and are commenced at various time points in pregnancy and delivered in an intense regimented or pragmatic manner. The physical activity-based interventions involved moderate exercise such as dancebased exercise programmes, water-based physical activity, stationery cycling, light intensity resistance training or enhanced routine daily activity, including walking. The mixed approach includes both diet and physical activity. Studies assessing other weight loss interventions such as pharmacotherapy or surgery will not be included.
We have previously identified the various interventions for inclusion in the IPD meta-analysis. ${ }^{1718}$ We will map the components and taxonomy of the interventions against the clinical outcomes.

\section{Outcome measures}

The primary outcomes are GDM as defined by NICE $^{19}$ or by any established criteria. Our secondary outcomes include other definitions of GDM (IADPSG, ${ }^{20}$ modified IADPSG, ${ }^{21}{ }^{22} \mathrm{WHO}^{23}$ and ADA criteria ${ }^{24}$ ), maternal and offspring complications such as hypertensive disorders, including pre-eclampsia, preterm birth, caesarean section and need for pharmacological therapy for hyperglycaemia, shoulder dystocia, respiratory distress syndrome, neonatal hypoglycaemia, stillbirth, neonatal death, perinatal death, Apgar score at 1 and $5 \mathrm{~min}$, birth weight, gestational age at birth, small/large for gestational age and admission to the neonatal intensive care unit (table 1). Subgroup analysis will explore whether a woman's BMI, age, parity, ethnicity and socioeconomic status modify the intervention effect.

\section{Study quality assessment and data collection}

The existing i-WIP dataset will be expanded to include new studies. We will use our previously peer-reviewed

Table 1 Structured research question

\section{Question \\ components}

\begin{tabular}{|c|c|}
\hline Population & Pregnant women with a BMI of $\geq 18.5 \mathrm{~kg} / \mathrm{m}^{2}$ in early pregnancy \\
\hline \multirow[t]{3}{*}{ Interventions } & Diet-based \\
\hline & Physical activity-based \\
\hline & Mixed approach: diet and/or physical activity with behavioural component \\
\hline \multirow[t]{7}{*}{ Outcomes } & Primary outcomes \\
\hline & $\begin{array}{l}\text { Gestational diabetes defined as per } 2015 \mathrm{NICE} \text { criteria (fasting glucose } 5.6 \mathrm{mmol} / \mathrm{L} \text { or above, and } 2 \text {-hour } \\
\text { glucose } 7.8 \mathrm{mmol} / \mathrm{L} \text { or above after a } 75 \mathrm{~g} \text { oral glucose tolerance test) }{ }^{19} \text { or GDM as defined within the study } \\
\text { by established criteria } \\
\text { Differential effects of interventions across subgroups based on maternal BMI, age, parity, ethnicity and } \\
\text { socioeconomic status }\end{array}$ \\
\hline & Secondary outcomes \\
\hline & Gestational diabetes with maternal and /or offspring complications \\
\hline & $\begin{array}{l}\text { Maternal: hypertensive diseases including pre-eclampsia, caesarean section, preterm birth and need for } \\
\text { pharmacological therapy for hyperglycaemia }\end{array}$ \\
\hline & $\begin{array}{l}\text { Offspring: shoulder dystocia, respiratory distress syndrome, neonatal hypoglycaemia, stillbirth, neonatal } \\
\text { death, perinatal death, Apgar score at } 1 \text { and } 5 \text { min, birth weight, gestational age at birth, small/large for } \\
\text { gestational age and admission to the neonatal unit } \\
\text { Categorisation of the interventions by core components and undertake network meta-analysis to rank } \\
\text { them by effectiveness }\end{array}$ \\
\hline & $\begin{array}{l}\text { Gestational diabetes as defined specifically using the IADPSG, }{ }^{20} \text { modified IADPSG, }{ }^{21}{ }^{22} \text { WHO }^{23} \text { and ADA } \\
\text { Cost-effectiveness of interventions using decision-analytical modelling }\end{array}$ \\
\hline $\begin{array}{l}\text { Study design of } \\
\text { included studies }\end{array}$ & Randomised trials \\
\hline
\end{tabular}


robust methods to assess the quality of the new studies and to extract and format the relevant data. ${ }^{12}$ We will use the risk of bias tool developed by the Cochrane Collaboration to score the quality of (the IPD from) each study. ${ }^{25}$ This will be based on study characteristics and supplemented with information from IPD when provided. Sensitivity analyses will examine the robustness of statistical and clinical conclusions to inform the inclusion or exclusion of trials deemed to be at high risk of bias.

We will extract additional data from existing and new studies where possible on the diagnostic criteria and oral glucose tolerance test (OGTT) values used to diagnose gestational diabetes. We will also extract relevant information on the intervention components, and offspring and maternal complications. We will follow the established, tested and trialled procedures used for data harmonisation in the i-WIP database. ${ }^{26}$ This will facilitate smooth and timely execution of the most time-consuming stage of the study, that is, data cleaning and its harmonisation.

\section{Sample size consideration}

Formal sample size calculations are not usually undertaken for meta-analyses. Brookes et al note that about four times the size of a single trial is required to detect an interaction with the same size as the overall treatment effect. ${ }^{27}$ We currently have access to the shared data for 24766 women (58 studies). The sample size has the potential to be increased further to 27538 women (71 studies) and beyond, depending on decisions to share data by other identified studies, and by the number of new studies published up to March 2021. We have undertaken a simulation-based approach to calculate the power of estimating genuine treatment-covariate interactions in our planned IPD meta-analysis, conditional on the number of trials, number of participants available in each trial and the covariate characteristics (eg, proportion of Caucasian subjects, mean and SD of BMI) while allowing for between-study heterogeneity in the treatment effect and the control group risk. ${ }^{28}{ }^{29}$ We calculated the power to detect a particular treatment-covariate interaction effect size in the subset of trials that report each covariate of interest (BMI, age, ethnicity, parity and socioeconomic status). Table 2 shows the power estimates, assuming an interaction between covariate and treatment effect corresponding to $30 \%$ (OR 0.70 ) and $25 \%$ reductions in gestational diabetes (OR 0.75 )

Table 2 Estimated power by simulation based on the IPD currently available in the i-WIP database*

\section{Assuming all 71 trials identified so far provide their IPD}

Trials (total
participants)
available for
the covariate

Covariate (subgroup) of interest

Estimated
power by
simulation
$(\%)$

For the $\mathbf{5 8}$ trials with IPD available/agreed

\begin{tabular}{ll}
\hline Trials (total & \\
participants) & Estimated \\
available for & power by \\
the covariate & simulation \\
(n) & $(\%)$
\end{tabular}

Assuming an interaction between covariate and treatment effect that corresponds to an OR of 0.70

\begin{tabular}{|c|c|c|c|c|c|}
\hline BMI & Obese versus non-obese & $70(27722)$ & 99.0 & $56(24443)$ & 97.6 \\
\hline Age & $\begin{array}{l}\text { Continuous assuming } \\
\text { linear trend }\end{array}$ & $69(27422)$ & 79.1 & $55(24143)$ & 78.4 \\
\hline Parity & $\begin{array}{l}\text { Nulliparous versus } \\
\text { multiparous }\end{array}$ & $59(22$ 253) & 95.4 & $48(19718)$ & 93.4 \\
\hline \multicolumn{6}{|c|}{ Assuming an interaction between covariate and treatment effect that corresponds to an OR of 0.75} \\
\hline BMI & Obese versus non-obese & 70 (27 722) & 92.8 & 56 (24 443) & 89.2 \\
\hline Age & $\begin{array}{l}\text { Continuous assuming } \\
\text { linear trend }\end{array}$ & 69 (27 422) & 72.4 & 55 (24 143) & 65.2 \\
\hline Ethnicity & $\begin{array}{l}\text { Caucasian versus non- } \\
\text { Caucasian }\end{array}$ & 48 (21 958) & 79.8 & 39 (19 394) & 74.4 \\
\hline Socioeconomic status & High versus low & $50(21136)$ & 85.4 & 41 (19 426) & 83.2 \\
\hline
\end{tabular}

${ }^{*}$ Assuming baseline risk of gestational diabetes is $11 \%$ on average, varying from $2 \%$ to $43 \%$ according to trial characteristics. BMI, Body Mass Index; IPD, individual participant data; i-WIP, International Weight Management in Pregnancy. 
across covariate (sub) groups, using the sample size of available IPD ( 58 trials), and also if all 71 trials shared their IPD. We expect to have sufficient events for the overall effect, and for most of the secondary outcomes with 5291 caesarean sections, 1154 preterm births; 1769 hypertensive diseases; 2483 large-for-gestational age babies; and 1420 babies admitted to the neonatal unit.

\section{Data analysis}

Overall effect and sub types of intervention

The effectiveness of the diet and physical activity-based interventions will be assessed using IPD meta-analytical framework. ${ }^{30}$ GDM as defined by NICE $^{19}$ or GDM as defined within the study by established criteria will be the main outcome. For each intervention type (all interventions, diet-based, physical activity-based and mixed approach), we will perform one-stage and two-stage IPD random-effect meta-analyses to obtain the pooled (summary) intervention effect on GDM via restricted maximum likelihood (ML) estimation or, for noncontinuous outcomes, ML estimation. One-stage models will use intercepts stratified by study to account for clustering, and covariates will be centred to improve estimation. ${ }^{31}$ CIs will be inflated to account for uncertainty in variance estimates (eg, using Hartung-Knapp and Kenward-Roger corrections for two-stage and one-stage approaches, respectively). ${ }^{32}$ All approaches will adjust for a few key prognostic factors available in all the IPD studies, which is anticipated to be age and BMI, to ensure conditional treatment effects are summarised. Adjustment factors will be stratified by study.

One-stage and two-stage analyses usually give similar results, and so any discrepancies will be resolved. ${ }^{33}$ We will use a random-effect meta-analysis approach, which allows for between-study heterogeneity in intervention effect, which is anticipated. Heterogeneity will be summarised using the I-squared statistic (which provides the proportion of total variability that is due to between-study heterogeneity) and the estimated between-study variance (" $\mathrm{au}^{2}$ "). To reveal the impact of heterogeneity more clearly, we will also calculate a $95 \%$ prediction interval for the intervention effect when applied in an individual clinical setting. The aforementioned analyses will also be undertaken for secondary outcomes, and GDM will be defined using other specific criteria such as IADPSG, ${ }^{20}$ modified IADPSG, ${ }^{21}{ }^{22} \mathrm{WHO}^{23}$ and ADA. ${ }^{24}$

\section{Differential effect by subgroups (treatment-covariate interactions)}

We will examine whether a woman's BMI, age, parity, ethnicity and socioeconomic status modify intervention effect. This will be undertaken by extending the two-stage and one-stage meta-analysis frameworks to include and then summarise treatment-covariate interaction terms, which provides the change in intervention effect for a 1-unit change in the covariate. We will always adjust for the prognostic effects of the covariate, in addition to others listed previously (ie, age and BMI). Ecological bias due to study-level confounding will be avoided using the deft approaches of Fisher et $a l^{44}$

Continuous variables will be kept as continuous to avoid arbitrary dichotomisation, and non-linear relationships and interactions will be modelled using restricted cubic splines and multivariate IPD meta-analysis. ${ }^{35}$ Subgroup analyses, if not carefully planned, can lead to misleading results, for example, due to the play of chance with multiple testing. ${ }^{25}$ Thus, caution will be used in interpretation of the collective set of subgroup results. However, our IPD meta-analysis will increase the power (often $>80 \%$ ) to detect genuine subgroup effects (treatmentcovariate interactions) and will also allow us to examine if there is consistency in the subgroup effect from study to study, rather than being a chance finding in a single study, for example.

\section{Examining potential sources of bias}

Small study effects (potential publication bias) will be investigated through the construction of contourenhanced funnel plots and appropriate statistical tests. To examine the impact of studies with unavailable IPD, we will extract (where available) appropriate aggregate study-level data (eg, interaction estimates) and incorporate them alongside the IPD using two-stage IPD random-effect meta-analysis. ${ }^{36}$ Sensitivity analyses will be undertaken that exclude studies not at low risk of bias, to ascertain if conclusions change when all studies are included. We will use the Grading of Recommendations, Assessment, Development and Evaluations approach to grade the evidence in our IPD meta-analysis. ${ }^{37}$

\section{Dealing with missing variables}

A range of strategies will be considered for dealing with missing data. In particular, multiple imputation will be used to impute partially missing variables within each study separately, under a missing at random assumption. If there are systematically missing variables then, where considered plausible, these will also be imputed by borrowing information across studies while allowing for heterogeneity and clustering in a multilevel imputation model. $^{38}$

\section{Decision-analytical modelling}

We will develop a decision-analytical simulation model to assess the cost-effectiveness of interventions involving diet and physical activity to prevent GDM and complications compared with usual care (control). The model will be developed using TreeAge Pro 2017 software (TreeAge Software, Williamstown, Massachusetts, USA). The structure and pathways will be informed by the data and trials included in the IPD meta-analysis, clinical input, NICE guidelines on the management of women in pregnancy and the approaches adopted in our previous model-based economic evaluations. ${ }^{12}$ The model will include all the potential pathways that could be followed by the women who will enter the model at the point of randomisation, to receive the intervention or care as usual. 
For the intervention effect, data from the IPD metaanalysis will estimate pooled effect ORs for the development of GDM. The baseline risk for the usual care group will be calculated on the pooled data for the control groups included in the trials. Maternal outcomes will not be considered where they are already observed at baseline. The estimated risk of maternal death will be from appropriate recent sources. ${ }^{39} 40$ NHS reference costs will provide much of the required cost data, and additional secondary sources will be interrogated. Costs from all secondary sources will be inflated as appropriate using the hospital and community health services pay and price index. ${ }^{41}$ Costs presented in foreign currency will be converted to UK pounds using historical annual average rates $^{42}$ and then inflated to current prices. Estimates of the cost of weight management interventions and antenatal and postnatal care costs will be based on results of our systematic review of economic evaluations of weight management interventions in pregnancy. ${ }^{12}$

The main analysis will compare costs and outcomes for a hypothetical cohort of 10000 pregnant women, based on the results of the IPD meta-analysis for all women. For secondary analysis, we will compare costs and outcomes for subgroups of women based on their characteristics, such as BMI, age, ethnicity, parity and socioeconomic status, to allow exploration of whether a lifestyle intervention in selective subgroups of women is a more costeffective strategy compared with care as usual. For all analyses, the relative cost-effectiveness of the intervention will be evaluated using effect size estimates from the IPD meta-analysis. An incremental approach will be adopted with a focus on the additional costs and benefits associated with a move from care as usual to diet and lifestyle interventions to manage weight gain in pregnancy. The results will be reported in terms of an incremental cost-effectiveness ratio of cost per unit of benefit gained, measured in natural clinical outcomes. The analysis will be conducted from the perspective of the health service (NHS), and only direct health service will be included. The time horizon adopted for both the primary and secondary analyses will be the start of pregnancy until the mother and infant are discharged from the hospital following the birth. Missing data will be addressed in the IPD meta-analysis.

We will perform Monte Carlo simulation to evaluate the effect of parameter uncertainty for probabilistic sensitivity analysis. We will summarise the results graphically using a cost-effectiveness acceptability curve to show the uncertainty surrounding the cost-effectiveness of the intervention for a range of thresholds for cost-effectiveness. A value of information analysis will be conducted to estimate the expected costs of uncertainty. Additionally, we will perform deterministic sensitivity analyses to check the robustness of the model. ${ }^{43}$ The expected cost of uncertainty is calculated by estimating the probability of making a wrong decision based on existing evidence, and the consequences of this wrong decision. The expected value of perfect information (EVPI) estimates the difference between the expected value of the decision made with perfect information and the decision made based on existing evidence. EVPI was calculated based on the methods described in Claxton and Posnett ${ }^{43}$. Among the many deterministic analyses to be explored, the effect of considering a longer time horizon will also be included.

\section{DISCUSSION}

We have previously shown in our IPD meta-analysis that women of all BMI groups could benefit from specific advice on diet and physical activity for weight gain in pregnancy. Findings from this proposal will show whether this benefit extends to gestational diabetes and other maternal or neonatal outcomes. Identification of the subset of women who would benefit from lifestyle interventions in pregnancy to prevent gestational diabetes will allow us to evaluate whether targeted management of these groups of women will improve their pregnancy outcomes. We will also be able to evaluate whether there is any differential effect according to the individual components of the intervention on pregnancy outcomes, which is required to provide a detailed recommendation. Our detailed protocol is produced in line with established guidance $^{15}$ that provides an in depth a priori overview of our IPD meta-analysis, which is intended to deliver implementable findings that can be translated into clinical practice and improve the care of women at risk of GDM and its associated complications.

The Delphi survey of our international collaborative group ranked GDM to be critically important to the care of pregnant women. ${ }^{44}$ In our previous IPD meta-analysis, we observed a statistically non-significant reduction in the rate of GDM with lifestyle interventions in our previous IPD meta-analysis (OR $0.89,95 \% \mathrm{CI} 0.72$ to 1.10$).{ }^{14}$ However, addition of aggregate data from studies that did not contribute IPD resulted in a stronger evidence of a significant reduction in gestational diabetes, but questions on effectiveness of such interventions on gestational diabetes remained due to the inherent bias with this approach. ${ }^{12}{ }^{14}$ The recent Cochrane review's aggregate meta-analysis showed similar findings ${ }^{13}$ and reported the quality of evidence to be low due to variations in the definitions, small sample sizes with imprecise estimates and statistical heterogeneity. However, the aforementioned reviews did not include the recently published large trials indicating potential beneficial effects of lifestyle intervention on gestational diabetes: the ESTEEM trial (1230 women, 2019) on Mediterranean diet reducing gestational diabetes, a component of the composite primary outcome, by $35 \%,{ }^{45}$ and the St Carlos trial ( 800 women), showing a similar reduction in gestational diabetes but with lower power. ${ }^{46}$

There are several other limitations with current evidence. First, the potential for lifestyle interventions to prevent GDM was only shown when aggregate and IPD data was combined and not in previous IPD meta-analysis alone. Second, whether the effects of diet and physical 
activity interventions would apply equally to all pregnant women or only to those at greater potential risk such as those with obesity, high maternal age or those from ethnic minorities origins is unknown. Third, the lack of detail about the interventions (including type, intensity and setting), as well as the associated costs of the interventions, limits delivery. Fourth, previous meta-analyses have been reliant on the definitions of GDM used in the original manuscript. Fifthly, the impact on clinically important outcomes, including complications of GDM, is not known.

We expect our proposed IPD meta-analysis to have additional power to identify differential effects across different risk groups of women on GDM risk as well as the components of the interventions in different patient groups that show clear benefits to prevent GDM. Currently, the key barriers to implementation of effective interventions to prevent gestational diabetes include lack of robust evidence on the effectiveness, lack of clarity about key components of complex diet and physical activity interventions, lack of information on costs associated with interventions and lack of information on who should be targeted. Our IPD meta-analysis will answer these questions. We have so far mapped the components of the interventions using the Intervention Description and Replication framework ${ }^{17}$ and categorised by type (diet, physical activity and mixed), frequency, intensity (high, medium and low), duration (prepregnancy, first trimester and second trimester), delivery (face-toface or e-health, individual and group sessions), setting (primary and secondary care), theoretical underpinning (eg, problem solving and action planning) and resources used. We also used a 96 -item framework ${ }^{18}$ to identify the behavioural change components applied within interventions. This work is crucial to our proposed plan to identify those components of the intervention that are most effective in preventing gestational diabetes.

Additionally, the incorporation of the raw data in the IPD meta-analysis will allow us to assess the impact of the interventions on GDM as defined by different criteria, including NICE, ${ }^{19}$ rather than a reliance on the definitions used by the original reporting paper. Following extensive collaboration with members of the i-WIP consortium, we have obtained additional data on all available OGTT values, which will be used in the proposed i-WIP GDM project. It is further anticipated that by using raw data, the breadth of outcomes that can be analysed will increase compared with aggregate meta-analysis; for example, papers may not have reported preterm delivery, yet raw data will incorporate the gestational age at delivery, allowing this outcome to be analysed.

Mutual trust and common research goal are the cornerstones of our i-WIP collaborative group. We have robust operating procedures in place for data access, publication and data sharing. The collaborative efforts need to take into account the equity challenges within systems, such as low socioeconomic status, deprivation and ethnicity, which are linked to variations in gestational diabetes rates and affect access to interventions. Organisational or delivery system-wide challenges depend on the setting, type of intervention, mode of delivery (digital, groups and individual-based) and workforce skills. An additional challenge is to rebalance the ongoing local, regional and national efforts and initiatives to tackle diabetes and obesity, with maternity transformation strategies focusing on improved maternal and child health, alongside efficient workforce skills development.

We will robustly address these by first identifying if the interventions need to be targeted to these groups and then by working with policymakers and patient and public involvement groups to identify ways to implement and reach communities that have the most need. Our work will directly feed into the ongoing efforts of NHS England to identify effective lifestyle interventions to prevent maternal obesity and complications like gestational diabetes. If specific subgroups of women were found to benefit the most, this can lead to re-organisation or commencement of new services in primary and secondary care. For for example, if women with obesity were found to benefit the most, current weight management services will need to incorporate the specific components of the intervention found to be most effective in preventing gestational diabetes. Furthermore, with increasing access to digital interventions, these findings could lead to the delivery of the intervention to virtual target groups. The cost-effectiveness findings and details on the type of effective intervention are key factors in implementation.

\section{ETHICS AND DISSEMINATION}

This is an evidence synthesis project involving metaanalysis of anonymised datasets. No further ethical considerations or approvals are needed. Guidance on participant data storage and management will be adhered to. The results will be published in peer-reviewed journals, shared in policy briefings to NHS England Directors, UK Chief Medical Officers, NICE Guideline leads, NHS providers and local authorities. We will also make our findings available in accessible formats to patient groups and relevant charities.

\section{Author affiliations}

${ }^{1}$ Institute of Applied Health Research, University of Birmingham, Birmingham, UK ${ }^{2}$ WHO Collaborating Centre for Global Women's Health, Institute of Metabolism and Systems Research, University of Birmingham, Birmingham, UK

${ }^{3}$ Maternity Dietetic Service, Women's Health Division, Barts Health NHS Trust, Antenatal Clinic, Royal London Hospital and Newham University Hospital, London, UK

${ }^{4}$ Centre for Exercise, Nutrition and Health Sciences, School for Policy Studies, University of Bristol, Bristol, UK

${ }^{5}$ Centre for Genomic Medicine and Child Health, Blizard Institute, Barts and the London School of Medicine and Dentistry, Queen Mary University of London, London, UK

${ }^{6}$ Population Health Sciences Institute, Newcastle University, Newcastle upon Tyne, UK

${ }^{7}$ Centre for Women's Health, Institute of Population Health Sciences, Queen Mary University of London, London, UK

${ }^{8}$ UNDP/UNFPA/UNICEF/WHO/World Bank Special Programme of Research, Development and Research Training in Human Reproduction, Department of Reproductive Health and Research, WHO, Geneva, Switzerland 
${ }^{9}$ Katie's Team Patient and Public Involvement Advisory Group, Queen Mary University of London, London, UK

${ }^{10}$ Department of Women and Children's Health, School of Life Course Sciences,

King's College London, London, UK

${ }^{11}$ Health Economics Unit, University of Birmingham, Birmingham, UK

${ }^{12} \mathrm{MRC} / \mathrm{CSO}$ Social and Public Health Sciences Unit, University of Glasgow, Glasgow, UK

${ }^{13}$ Monash Centre for Health Research and Implementation, School of Public Health,

Monash University, Melbourne, Victoria, Australia

${ }^{14}$ Centre for Prognosis Research, School of Medicine, Keele University, Keele, UK

${ }^{15}$ Pragmatic Clinical Trials Unit, Institute of Population Health Sciences, Barts and the London, School of Medicine and Dentistry, Queen Mary University of London, London, UK

${ }^{16}$ Birmingham Women's and Children's NHS Foundation Trust, Birmingham, UK

Twitter Jonathan M Hazlehurst @hazlehurst_j and Tracy Roberts @ tracyrobertsbham

Collaborators International Weight Management in Pregnancy (i-WIP) Collaborative Group.

Contributors ST and JA conceptualised the protocol and acted as guarantors of the review. DC, JMH, ST and JA wrote the manuscript with significant critical input from FA, CF, GH, NH, SI, APB, NM, LP, KN, TR, SAS, HJT and RR. RR provided statistical input. All authors reviewed the manuscript, contributing critical changes and approved the final version of the manuscript.

Funding NIHR Health Technology Assessment Programme (NIHR129715).

Competing interests None declared.

Patient consent for publication Not required.

Provenance and peer review Not commissioned; peer reviewed for ethical and funding approval prior to submission.

Supplemental material This content has been supplied by the author(s). It has not been vetted by BMJ Publishing Group Limited (BMJ) and may not have been peer-reviewed. Any opinions or recommendations discussed are solely those of the author(s) and are not endorsed by BMJ. BMJ disclaims all liability and responsibility arising from any reliance placed on the content. Where the content includes any translated material, BMJ does not warrant the accuracy and reliability of the translations (including but not limited to local regulations, clinical guidelines, terminology, drug names and drug dosages), and is not responsible for any error and/or omissions arising from translation and adaptation or otherwise.

Open access This is an open access article distributed in accordance with the Creative Commons Attribution Non Commercial (CC BY-NC 4.0) license, which permits others to distribute, remix, adapt, build upon this work non-commercially, and license their derivative works on different terms, provided the original work is properly cited, appropriate credit is given, any changes made indicated, and the use is non-commercial. See: http://creativecommons.org/licenses/by-nc/4.0/.

ORCID iD

Jonathan M Hazlehurst http://orcid.org/0000-0003-1036-103X

\section{REFERENCES}

1 American Diabetes Association. 2. Classification and Diagnosis of Diabetes: Standards of Medical Care in Diabetes-2018. Diabetes Care 2018;41:S13-27.

2 Hyperglycaemia in pregnancy (hip) (20-49 Y): prevalence of gestational diabetes mellitus (GDM) \%. Available: https:// diabetesatlas.org/data/en/indicators/14/ [Accessed 10 Sep 2020].

3 Waters TP, Dyer AR, Scholtens DM, et al. Maternal and neonatal morbidity for women who would be added to the diagnosis of GDM using IADPSG criteria: a secondary analysis of the hyperglycemia and adverse pregnancy outcome study. Diabetes Care 2016;39:2204-10.

4 Gillespie P, Cullinan J, O'Neill C, et al. Modeling the independent effects of gestational diabetes mellitus on maternity care and costs. Diabetes Care 2013;36:1111-6.

5 Xu T, Dainelli L, Yu K, et al. The short-term health and economic burden of gestational diabetes mellitus in China: a modelling study. BMJ Open 2017;7:e018893.

6 Vounzoulaki E, Khunti K, Abner SC, et al. Progression to type 2 diabetes in women with a known history of gestational diabetes: systematic review and meta-analysis. BMJ 2020;369:m1361.
7 Hammoud NM, Visser GHA, van Rossem L, et al. Long-Term BMI and growth profiles in offspring of women with gestational diabetes. Diabetologia 2018;61:1037-45.

8 Kawasaki M, Arata N, Miyazaki C, et al. Obesity and abnormal glucose tolerance in offspring of diabetic mothers: a systematic review and meta-analysis. PLoS One 2018;13:e0190676.

9 Walker J, Colhoun H, Livingstone S, et al. Type 2 diabetes, socioeconomic status and life expectancy in Scotland (20122014): a population-based observational study. Diabetologia 2018;61:108-16.

10 Holmes J, McGill S, Kind P, et al. Health-Related quality of life in type 2 diabetes (T2ARDIS-2). Value in Health 2000;3:S47-51.

11 Hex N, Bartlett C, Wright D, et al. Estimating the current and future costs of Type 1 and Type 2 diabetes in the UK, including direct health costs and indirect societal and productivity costs. Diabet Med 2012;29:855-62.

12 Rogozińska E, Marlin N, Jackson L, et al. Effects of antenatal diet and physical activity on maternal and fetal outcomes: individual patient data meta-analysis and health economic evaluation. Health Technol Assess 2017;21:1-158.

13 Shepherd E, Gomersall JC, Tieu J, et al. Combined diet and exercise interventions for preventing gestational diabetes mellitus. Cochrane Database Syst Rev 2017;111.

14 International Weight Management in Pregnancy (i-WIP) Collaborative Group. Effect of diet and physical activity based interventions in pregnancy on gestational weight gain and pregnancy outcomes: meta-analysis of individual participant data from randomised trials. BMJ 2017;358:j3119.

15 Moher D, Shamseer L, Clarke M, et al. Preferred reporting items for systematic review and meta-analysis protocols (PRISMA-P) 2015 statement. Syst Rev 2015;4:1.

16 Stewart LA, Clarke M, Rovers M. Preferred reporting items for a systematic review and meta-analysis of individual participant data: the PRISMA-IPD statement. JAMA - J. Am. Med. Assoc 2015.

17 Hoffmann TC, Glasziou PP, Boutron I, et al. Better reporting of interventions: template for intervention description and replication (TIDieR) checklist and guide. BMJ 2014;348:g1687.

18 Michie S, van Stralen MM, West R. The behaviour change wheel: a new method for characterising and designing behaviour change interventions. Implementation Sci 2011;6:42.

19 National Institute for Health and Care Excellence. Diabetes in pregnancy : management from preconception to the postnatal period. NICE 2015 .

20 International Association of Diabetes in Pregnancy Study Group. Recommendations on the diagnosis and classification of hyperglycemia in pregnancy. In: Diabetes Care, 2010.

21 Poston L, Bell R, Croker H. Effect of a behavioural intervention in obese pregnant women (the upbeat study): a multicentre, randomised controlled trial. Lancet Diabetes Endocrinol 2015.

22 Al Wattar BH, Dodds J, Placzek A, et al. Effect of simple, targeted diet in pregnant women with metabolic risk factors on maternal and fetal outcomes (Esteem): study protocol for a pragmatic multicentre randomised trial. BMJ Open 2016;6:e013495.

23 WHO. World Health organization: definition and diagnosis of diabetes mellitus and intermediate hyperglycemia. Geneva: World Health Org. WHO2, 2006.

24 Goyal A, Gupta Y, Singla R, et al. American Diabetes Association "Standards of Medical Care-2020 for Gestational Diabetes Mellitus": A Critical Appraisal. Diabetes Ther 2020;11:1639-44.

25 Cochrane Handbook for systematic reviews of interventions 2019.

26 i-WIP Collaborative Group. Centro Rosarino de Estudios Perinatales. i-WIP. Repository of individual participant data from randomised controlled trials with diet and physical activity based interventions in pregnancy, 2013. Available: https://kamolo.org/iwipipd/ [Accessed 7 Dec 2020].

27 Brookes ST, Whitley E, Peters TJ, et al. Subgroup analyses in randomised controlled trials: quantifying the risks of false-positives and false-negatives. Health Technol Assess 2001;5.

28 Kontopantelis E, Springate DA, Parisi R, et al. Simulation-Based Power Calculations for Mixed Effects Modeling: ipdpower in Stata. J Stat Softw 2016;74:1-25.

29 Ensor J, Burke DL, Snell KIE, et al. Simulation-Based power calculations for planning a two-stage individual participant data meta-analysis. BMC Med Res Methodol 2018;18:41.

30 Ruifrok AE, Rogozinska E, van Poppel MNM, et al. Study protocol: differential effects of diet and physical activity based interventions in pregnancy on maternal and fetal outcomes-individual patient data (IPD) meta-analysis and health economic evaluation. Syst Rev 2014;3.

31 Riley RD, Legha A, Jackson D, et al. One-stage individual participant data meta-analysis models for continuous and binary outcomes: 
comparison of treatment coding options and estimation methods. Stat Med 2020;39:2536-55.

32 Cornell JE, Mulrow CD, Localio R. Random-effects meta-analysis of inconsistent effects: a time for change. Ann Intern Med 2014

33 Burke DL, Ensor J, Riley RD. Meta-Analysis using individual participant data: one-stage and two-stage approaches, and why they may differ. Stat Med 2017

34 Fisher DJ, Carpenter JR, Morris TP, et al. Meta-analytical methods to identify who benefits most from treatments: daft, deluded, or deft approach? BMJ 2017;356:j573.

35 Riley RD, Debray TPA, Fisher D, et al. Individual participant data meta-analysis to examine interactions between treatment effect and participant-level covariates: statistical recommendations for conduct and planning. Stat Med 2020;39:2115-37.

36 Ahmed I, Sutton AJ, Riley RD. Assessment of publication bias, selection bias, and unavailable data in meta-analyses using individual participant data: a database survey. BMJ 2011;344:d7762.

37 Guyatt GH, Oxman AD, Vist GE, et al. Grade: an emerging consensus on rating quality of evidence and strength of recommendations. $B M J$ 2008;336:924-6.

38 Quartagno M, Grund S, Carpenter J. jomo: a flexible package for two-level joint modelling multiple imputation. $R \mathrm{~J} 2019$.

39 Regan L, Rai R. Epidemiology and the medical causes of miscarriage. Bailliere's Best Pract Res Clin Obstet Gynaecol.
40 Knight M, Kenyon S, Brocklehurst $\mathrm{P}$, et al. on behalf of $\mathrm{M}-\mathrm{U}$. Saving Lives, Improving Mothers' Care Lessons learned to inform future maternity care from the UK and Ireland Confidential Enquiries into Maternal Deaths and Morbidity 2009-2012 - UCL Discovery. Report 2014.

41 Department of Health. National schedule of reference costs: main schedule 2015 to 2016. NHS Ref. costs 2015 to 20162016 .

42 UKForex - foreign exchange services.

43 Claxton K, Posnett J. An economic approach to clinical trial design and research priority-setting. Health Econ 1996;5:513-24.

44 Rogozinska E, D'Amico MI, Khan KS, et al. Development of composite outcomes for individual patient data (IPD) meta-analysis on the effects of diet and lifestyle in pregnancy: a Delphi survey. BJOG: Int J Obstet Gy 2016;123:190-8.

45 Al Wattar B H, Dodds J, Placzek A. Mediterranean-style diet in pregnant women with metabolic risk factors (Esteem): a pragmatic multicentre randomised trial. PLoS Med 2019.

46 Assaf-Balut C, García de la Torre N, Durán A, et al. A Mediterranean diet with additional extra virgin olive oil and pistachios reduces the incidence of gestational diabetes mellitus (GDM): a randomized controlled trial: the St. Carlos GDM prevention study. PLoS One 2017; 12:e0185873. 